\section{A computerized approach for determining equal appearing interval attitude scales}

\author{
RICHARD J. HOFMANN \\ Miami University, Oxford, Ohio 45056
}

The objectives of this brief article are to: (a) report on a method for constructing attitude scales following the equal appearing interval ideas of Thurstone and Chave (1929, pp. 23-58) and (b) discuss a computer program to be used with this method.

Thurstone and Chave asked their subjects to sort a large number of statements into 11 piles. In sorting the statements the subjects were asked not to express their own opinions, rather they were asked to judge the degree of favorableness of a statement according to a theoretical scale from 0 to 11 . To adapt this procedure to a computer, an IBM 555 response sheet was utilized. This response sheet is unique in that it provides for 10 response categories. Rather than sorting the statements into 11 piles, with a neutral 6th pile, the computerized approach technically eliminates a neutral category, which forces the subject to sort into either Categories 0 through 4 or Categories 5 through 9 , resulting in a theoretical neutral category of 4.5. Subjects received a set of instructions which corresponded very closely to the instructions provided by Thurstone and Chave. However, the instructions were oriented toward recording responses on an IBM 555 response sheet.

After the IBM 555 response sheets had been completely filled out, they were processed and converted by machine, an IBM 1232 optical pagereader, to an 80-column Hollerith card. Inasmuch as each IBM 555 form has 10 response categories for each of 150 items, and inasmuch as each item is associated with a single column of a Hollerith card, there was a

This program was developed and written while the author was a faculty research fellow at Miami University. maximum of $17 / 8$ cards. These cards were then utilized in the analysis of the statements.

Analysis of Hollerith Cards. In processing the cards, the response frequency and cumulative proportions for each of the 10 response categories were determined for each item. The scale value was determined for each item. This value ranged from a low of unity to a high of 10 , with a value of 5.5 representing a neutral item. The scale was indicative of an item's relative placement on an assumed bipolar continuum. A scale value of unity was associated with an extremely positive statement. Also included for each item was an index which was indicative of the item's ambiguity, an ambiguity value.

The average ambiguity value was computed for each unit of scale value. This average was reported in tabular form along with a frequency count of the number of statements defined within each unit of scale value. Finally the standard error of a scale value was determined.

Program Capacity. The program requires 8,118 bytes of core as it is presently written. Presently programmed in FORTRAN IV to accommodate 50 items, with up to 10 response categories, the capacity is easily adjusted by changing one statement in the program. Using an IBM 370-168 G-level compiler, it required approximately $1 \mathrm{sec}$ to compile and run a test problem of 32 items, 10 response categories, and 190 subjects.

Availability. Copies of this article, an illustrative set of directions, an IBM 555 form, a source listing, and documented output from an illustrative example can be obtained without charge by writing to Richard J. Hofmann, Department of Education Psychology, Miami University, Oxford, Ohio 45056.

\section{REFERENCES}

Thurstone, L. L., \& Chave, E. J. The measurement of attitude: A psychophysical method and some experiments with a scale for measuring attitude toward the church. Chicago: The University of Chicago Press, 1929. Pp. 23-58. 